\title{
Enteropathogenicity of Dutch and German avian reoviruses in SPF white leghorn chickens and broilers
}

\author{
Thaweesak SONGSERM ${ }^{\mathrm{a}, \mathrm{b}}$, Dirk van RoOZELAAR ${ }^{\mathrm{a}}$, Arie KANT $^{\mathrm{a}}$, Jan POL $^{\mathrm{a}}$, \\ Anton PIJPERS ${ }^{\mathrm{b}, \mathrm{c}}$, Agnes TER HUURNE $^{\mathrm{a} *}$ \\ ${ }^{a}$ ID-Lelystad BV, Institute for Animal Science and Health, PO Box 65, 8200 AB, Lelystad, \\ The Netherlands \\ ${ }^{\mathrm{b}}$ Department of Farm Animal Health, Faculty of Veterinary Medicine, Utrecht University, \\ Yalelaan 7, $3584 \mathrm{CL}$, Utrecht, The Netherlands \\ ${ }^{c}$ Animal Health Service, PO Box 9, 7400 AA Deventer, The Netherlands
}

(Received 5 April 2001; accepted 5 December 2002)

\begin{abstract}
The enteropathogenicity of avian reoviruses (ARVs), isolated from chickens affected with malabsorption syndrome (MAS) from The Netherlands and Germany was studied. In the first trial seven different ARVs isolated from MAS cases were inoculated in 1-day-old specific pathogenic free (SPF) white leghorns. The pathogenicity was compared with 2 ARVs isolated from cases of tenosynovitis, namely reference strain S1133 and a Dutch strain. Although a difference in the severity of the clinical disease was observed, all reoviruses could induce vacuolar degeneration and sloughing of the epithelium of the small intestine at day 2 post inoculation (PI) till day 7 PI. Two Dutch and one German ARV derived from MAS causing the most severe intestinal lesions at day 2 PI, were further studied in the second trial using SPF broilers. These reoviruses did not cause weight gain depression in the broilers although lesions in the small intestine were present from day 1 up to day 4 PI and were more severe than in the white leghorn chickens. In one of the inoculated groups apical denuded villi were already present at day 1 PI. At day 7 PI the small intestine of the infected broilers appeared to be normal. Reovirus antigen was detected in the cytoplasm of the enterocytes at the tip and middle section of the affected villi both in layers and in broilers. To study the role of intestinal $\mathrm{CD}^{+}$and $\mathrm{CD} 8^{+} \mathrm{T}$-cells and macrophages/monocytes in the pathogenesis of ARV, the numbers of these cells of the jejunal villi of one infected and the control broiler groups were compared. $\mathrm{CD} 4{ }^{+} \mathrm{T}$-cells were detected in low numbers and only in the infected broiler group at day 14 PI. The numbers of $\mathrm{CD}^{+} \mathrm{T}$-cells and macrophages/monocytes were significantly higher in the infected broiler group than in the control broiler group at day 7 and 14 PI and at day 7 PI respectively. Our study indicates that the reovirus alone cannot induce intestinal lesions as found in MAS chickens. Moreover, $\mathrm{CD} 8^{+} \mathrm{T}$-cells may play a major role in the pathogenesis and or reovirus clearance in the small intestine.
\end{abstract}

malabsorption syndrome / runting-stunting syndrome / reovirus / enteropathogenicity / chicken

\footnotetext{
* Correspondence and reprints

Tel.: (31) 320-238264; fax: (31) 320-238094; e-mail: agnesterhuurne@planet.nl
} 


\section{INTRODUCTION}

The malabsorption syndrome (MAS) or runting-stunting syndrome is an economic problem in the broiler industry and is distributed around the world. Commonly, the susceptible age to MAS is the first two weeks of age. This syndrome is mainly characterised by gastrointestinal lesions directly resulting in weight gain depression. The gastrointestinal lesions generally include proventriculitis and enteritis with cellular infiltration, cystic crypts of Lieberkühn, villus atrophy and villus fusion [19]. Avian reoviruses (ARVs) have been associated with MAS, both in field surveys [5, 6, 12, 27] and in controlled experiments $[11,22]$. ARVs are also associated with disease conditions like tenosynovitis [3, 25]. Although the relationship between reovirus and tenosynovitis is well-established [25], this role is less clear in MAS. Reoviruses isolated from MAS chickens could induce lesions/microscopic changes in the liver [24], heart [17], bursa of Fabricius [15, 17], bone [27], proventriculus, intestine and tendons [20]. Reoviruses associated with tenosynovitis could also induce intestinal lesions [21].

Recently, we have shown reovirus antigens in lesions of the small intestine of MAS affected broilers early after inoculation of the MAS-homogenates [22]. Therefore we hypothesise that the reovirus may be a priming agent in the early development of the intestinal lesions in cases of MAS.

The role that different ARVs isolated from MAS play in the intestinal lesions and weight gain depression needs to be further investigated. This paper describes the enteropathogenicity of reoviruses isolated in a controlled MAS experiment [22] and from field cases of MAS in The Netherlands and Germany. In the first trial, we compared the enteropathogenicity of several MAS- associated reoviruses in SPF white leghorn chickens with that of two tenosynovitis-related ARVs by histopathology. In the second trial, the entero- pathogenicity of MAS-associated ARVs is studied in 1-day-old SPF broilers.

\section{MATERIALS AND METHODS}

\subsection{Chickens}

One-day-old specific pathogen free (SPF) white leghorn chicks and SPF broiler chicks were used in trial 1 or 2 respectively. The SPF white leghorn chickens were obtained from the Institute for Animal Science and Health, Lelystad, The Netherlands. The SPF broiler chickens were obtained from the Animal Health Service, Deventer, The Netherlands. The broiler chickens are a crossbred of Hybro and Cobb. Neither SPF white leghorn nor SPF broiler chickens had maternal antibodies against the reovirus and both were reovirus-negative upon isolation procedures.

\subsection{Viruses}

Reovirus isolates used in trial 1 are presented in Table I. The MAS-associated ARVs used were obtained from 2 sources. ARV GEI10 97M, NLI01 80M, NLI02 88M, GEI09 97M, and NLI12 96M were isolated from MAS-homogenates used in the former study [22]. ARV NLI20 98M and NLI19 97M were isolated from the intestinal contents of MAS affected chickens in the field. We also used two tenosynovitis-associated ARVs: S1133 [26] and a Dutch field strain NLA13 96T, isolated at the Animal Health Service, Deventer, The Netherlands. All ARVs were propagated in chicken embryo liver cells (CELC). The fourth passage on CELC was used for inoculation. The viruses were identified as reoviruses with the immunoperoxidase monolayer assay (IPMA) as described earlier [1] using rabbit anti-reovirus polyclonal antibodies. All viruses were genotyped based on the $\sigma \mathrm{C}$ amino acid sequence and were classified in clusters [10]. (Clusters are indicated in Tab. I.) 
Table I. Enteropathogenicity of avian reoviruses with a different origin and genotype inoculated in 1-day-old SPF White Leghorn chicks (trial 1).

\begin{tabular}{|c|c|c|c|}
\hline $\begin{array}{l}\text { Group, } \\
\text { reovirus code }^{\text {a }}\end{array}$ & $\begin{array}{l}\text { Genotype } \\
\text { cluster }^{\mathrm{a}}\end{array}$ & $\begin{array}{c}\text { GenBank } \\
\text { accession number }{ }^{b}\end{array}$ & $\begin{array}{l}\text { Number of affected } \\
\text { birds at day } 2 \mathrm{PI}(n=5)\end{array}$ \\
\hline 1, PBS (control) & - & & 0 \\
\hline 2, NLI01 $80 \mathrm{M}^{\mathrm{d}}$ & 4 & AF 354195 & 2 \\
\hline 3, NLI02 88M $\mathrm{M}^{\mathrm{d}}$ & 4 & AF354229 & 1 \\
\hline 4, NLI12 $96 \mathrm{M}^{\mathrm{d}}$ & 4 & AF354230 & 1 \\
\hline 5, NLI20 98M & 2 & AF354197 & 4 \\
\hline 6, NLI19 97M & 4 & AF354196 & 3 \\
\hline 7, GEI10 97M $\mathrm{M}^{\mathrm{d}}$ & $1,4,5$ & AF354119 & 2 \\
\hline 8, GEI09 97M ${ }^{\mathrm{d}}$ & $1,4,5$ & AF354220 & 3 \\
\hline 9, NLA13 96T & 4 & AF354228 & 1 \\
\hline 10, S1133 [25] & 1 & & 2 \\
\hline
\end{tabular}

\footnotetext{
${ }^{\mathrm{a}}$ Reovirus codes and genotype clusters, according to Kant et al. [10]

Designation: NL = case in The Netherlands; I = isolated at ID-Lelystad; $01=$ first isolate of the year;

80 = year of isolation; $M=$ MAS originating. Furthermore: GE = Germany; A = isolate from Animal

Health Service, Deventer; T = tenosynovitis; PBS = phosphate buffered saline.

${ }^{b}$ GenBank accession numbers of $\sigma \mathrm{C}$ sequences as submitted by Kant et al. [10].

${ }^{\mathrm{c}}$ Histopathological changes include vacuolar degeneration of the epithelium and sloughing of the epithelium at the tip of the villi.

${ }^{\mathrm{d}}$ Reoviruses obtained from a former MAS study [22].
}

Since Kant et al. [10] have submitted these sequence data to GenBank, the accession numbers are indicated in Table I.

Based on the results in SPF layers in trial 1, it was decided to carry out an enteropathogenicity test in SPF broilers with a selection of the formerly used reoviruses: GEI09 97M, NLI19 97M, and NLI20 98M. Since in the mean time, it was found that the GEI10 97M contained 3 genotypes (clusters 1, 4, 5 in Kant et al. [10]), this virus was cloned by the limiting dilution method. Before use in the broilers, ARVs NLI19 97M, NLI20 98M and also one clone of GEI09 97M (genotype cluster 4, Kant et al. [10]) were cloned. The passage number of these clones on CELC used for inoculation into the broilers was 7, 7 and 12 respectively. We also used ARV GEI09 97M containing all 3 genotypes (fourth passage on CELC).

All ARVs used in both trials were titrated for a $50 \%$ tissue culture infective dose $\left(\mathrm{TCID}_{50}\right)$ before inoculation into the chickens.

\subsection{Experimental designs}

\subsubsection{Trial 1: Enteropathogenicity test in SPF white leghorn}

One hundred 1-day old SPF white leghorn chicks were divided into 10 groups, 10 chicks each. Group 1 was orally inoculated with $0.5 \mathrm{~mL}$ Dulbecco phosphate buffered saline (PBS) and served as a control group. Groups 2 to 10 were orally inoculated with $0.5 \mathrm{~mL}$ of a suspension containing $10^{6} \mathrm{TCID}_{50} / \mathrm{mL}$ PBS of NLI01 80M, NLI02 88M, NLI12 96M, NLI20 98M, NLI19 97M, GEI10 97M, GEI09 97M, NLA13 96T and S1133, respectively (Tab. I). The chickens were kept in isolators and given feed and water ad libitum.

The chicks were killed and necropsied on days 2 and 7 post infection (PI). Pieces 
Table II. Effect of inoculation of 1-day-old SPF broilers with different avian reoviruses on body weight.

\begin{tabular}{llcc}
\hline \multirow{2}{*}{$\begin{array}{l}\text { Group, } \\
\text { reovirus code (cluster) }\end{array}$} & \multicolumn{3}{c}{ Body weight in grams (means \pm SD) $(n=5)$} \\
\cline { 2 - 4 } & day 7 PI & day 14 PI & Day 21 PI \\
\hline 1 Control, PBS & $84 \pm 16.1$ & $290 \pm 17.1$ & $517 \pm 30.1^{\mathrm{a}, \mathrm{c}}$ \\
2 GEI09 97M $(1,4,5)$ & $85 \pm 6.3$ & $290 \pm 37.8$ & $460 \pm 50.9^{\mathrm{a}}$ \\
3 GEI09 97M (4) & $82 \pm 7.8$ & $255 \pm 22.1$ & $590 \pm 65.6^{\mathrm{b}}$ \\
4 NLI19 97M (4) & $90 \pm 8.8$ & $268 \pm 26.8$ & $545 \pm 49.8^{\mathrm{b}, \mathrm{c}}$ \\
5 NLI20 98M (2) & $84 \pm 12.5$ & $275 \pm 39.4$ & $527 \pm 35.8^{\mathrm{b}, \mathrm{c}}$ \\
\hline
\end{tabular}

${ }^{\mathrm{A}}$ Reovirus code and cluster according to genotyping by Kant et al. [10]. Designation: GE $=$ case in Germany; I = isolated at ID-Lelystad; $09=9$ th isolate of the year; $97=$ year of isolation; M = MAS originating. Furthermore: $\mathrm{NL}=$ The Netherlands; $\mathrm{PBS}=$ phosphate buffered saline.

a,b,c Means \pm SD in a column with no common superscript differ significantly $(P<0.05)$.

of the proventriculus, duodenum, jejunum, ileum, cecal tonsil and bursa of Fabricius were fixed in $10 \%$ buffered formalin and processed for a histopathological study. Parts of the same organs were snap frozen in liquid nitrogen and kept at $-70{ }^{\circ} \mathrm{C}$ for immunohistochemistry.

\subsubsection{Trial 2: The enteropathogenicity test in SPF broilers}

One hundred and seventy-five 1-day-old SPF broiler chicks were divided into 5 groups, 35 chicks each. Group 1 was orally inoculated with $0.5 \mathrm{~mL}$ PBS and served as a control group. Groups 2 to 5 were orally inoculated with $0.5 \mathrm{~mL}$ of viral suspension containing $10^{6} \mathrm{TCID}_{50} / \mathrm{mL}$ PBS of GEI09 97M (containing 3 genotypes), cloned GEI09 97M (cluster 4), cloned NLI19 97M (cluster 4) and cloned NLI20 98M (cluster 2), respectively (Tab. II). Five chickens per group were weighed on days 7, 14 and 21 PI and necropsied on days 1, 2, 4, 7 and 14 PI, whereas on days 21 PI the remaining chickens $(n=$ 10 per group) were weighed and necropsied. Pieces of the proventriculus, duodenum, jejunum, ileum, pancreas, cecal tonsil and bursa of Fabricius were fixed with $10 \%$ buffered formalin for a histopathological study. The same parts of the intestine were snap frozen in liquid nitrogen and kept at $-70{ }^{\circ} \mathrm{C}$ for immunohistochemistry.

Cloacal swabs were taken for virus isolation at day 7 PI. Blood was collected before inoculation $(n=10)$ and at day $21 \mathrm{PI}$ $(n=10)$ via heart puncture for separation of the serum to test for anti-reovirus antibodies.

\subsection{Histopathology and immunohistochemistry}

All formalin-fixed tissues were processed according to standard procedures and stained with haematoxylin and eosin (H\&E) [14]. The slides were studied under a light microscope. All frozen tissues were sectioned on a cryostat at $8-\mu \mathrm{m}$ thickness and taken up on glass slides (Superfrost ${ }^{\mathrm{B}}$; BDH, Poole, England). The tissues were fixed with pure acetone and air-dried. An immunoperoxidase staining was performed for detection of reovirus antigen in the small intestine, cecal tonsil and bursa of Fabricius as described earlier [22]. To study the intestinal cellular immune response in broilers after reovirus infection, an immunoperoxidase staining was performed on cryostat tissues of the jejunum of broilers in group 5 (reovirus NLI20 98M) and group 1 (control) in trial 2. 
Monoclonal antibodies anti-macrophage/ monocyte [7], helper T-cells $\left(\mathrm{CD}^{+}\right)$and cytotoxic T-cells $\left(\mathrm{CD}^{+}\right)$(Southern Biotechnology Associates, Inc, Birmingham, AL, USA) were used separately. The numbers of positive cells (pc) in the villi were assessed by randomly measuring the cut surface area of longitudinal sections of the villi that did not contain germinal centres or lymphocytic aggregations. A cut surface area (in square micron, sm) of a villus was measured by the Optimas 5.2 Image Analysis System (Washington DC, USA). The cut surface area of a villus was measured from one side of the base of the villus (lamina propria), passing the rim, the top of the villus until the other side of the base of the villus. To measure the cut surface area of the villus, an optical magnification of 100 times was used. After measuring the cut surface area, the positive cells in this area were counted by a hand-counter with optical magnification of 250 times or higher in order to get a clear boundary of a positive cell. This procedure was done for 3 sections of the jejunum per chick and for 3 villi per section. Hence the cut surface area and the number of positive cells were assessed 9 times in one chicken.

\subsection{Virus re-isolation}

All swab samples from the inoculated and the control broilers of the experiment were tested by virus isolation. The samples were diluted in PBS, and inoculated onto primary CELC. Inoculated cell cultures were observed for a typical cytopathic effect (CPE) for 4 days. Samples that were CPE-negative in the first passage were blindly passed at least 3 times. Isolation of the reovirus was confirmed by IPMA at 24-48 hours PI, as mentioned in 2.2.

\subsection{Serology}

All sera collected before inoculation and sera collected at day 21 PI were tested for antibodies against the reovirus by IPMA.

\subsection{Statistics}

Data were analysed with a two-way analysis of variance model according to SPSS for windows Release 6.1, 1994 [23]. The level of significance used was $P<$ 0.05 .

\section{RESULTS}

\subsection{Enteropathogenicity test in SPF white leghorn in (trial 1)}

The infected chicks had varying clinical signs and gross and histopathological changes. A few chicks in groups 2, 4, 5, 8 and 9 showed weakness and mild diarrhoea at day 2 PI. At day 7 PI, no clinical signs were seen. Macroscopically, some chicks of these groups had distended and pale small intestines with watery and mucous contents. The numbers of affected chickens at day 2 PI are presented in Table I. Histopathological changes including vacuolar degeneration of the epithelium and sloughing of the epithelium were present at the tip of the villi. Although the lesions were also present in the duodenum, they were the most prominent in the jejunum and ileum. Also, these small intestinal lesions were most obvious in groups 5, 6 and 8 (infected with MAS-associated ARVs NLI20 98M, NLI19 97M and GEI09 97M), whereas the lesions were less severe in chickens in groups 9 and 10 (infected with tenosynovitis-associated strains, NLA13 96T and S1133). At day 7 PI, all infected groups showed more or less severe lesions in the small intestines including vacuolar degeneration of the epithelium and sloughing of the tip of the villi. The lesions were, however, so straight that part of the lesions might be artificial because of the handling of the intestines. No histopathological changes were present in the control group at either time point. 


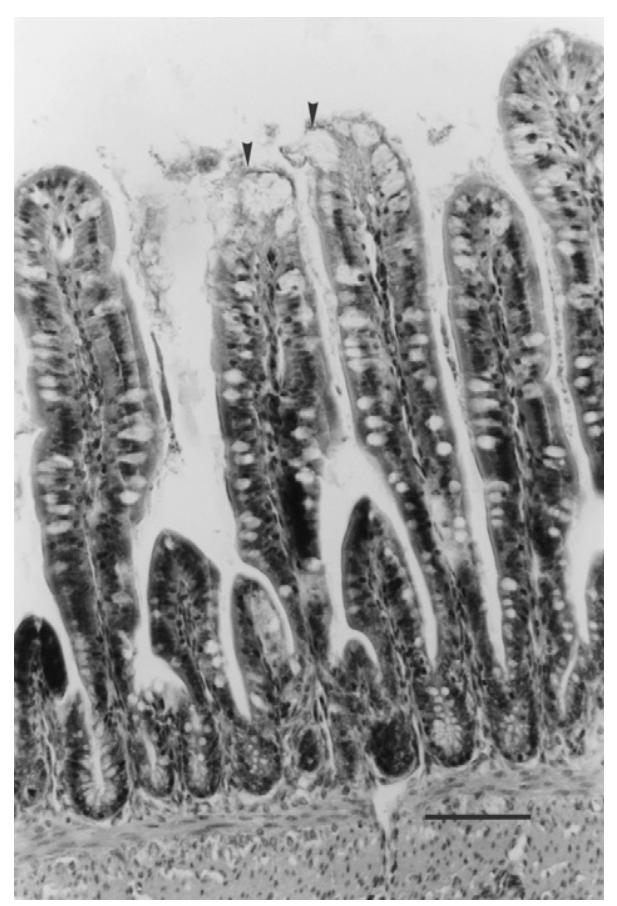

Figure 1. Infected intestinal villi of the ileum from a SPF broiler infected with ARV NLI20 $98 \mathrm{M}$. Vacuolar degeneration and sloughing of the villus epithelium (arrow head) at day 2 PI. Bar $=105 \mu \mathrm{m}$.

\subsection{An enteropathogenicity test in SPF broilers (trial 2)}

\subsubsection{Clinical signs, gross lesions, histological changes and immunohistochemistry}

None of the ARVs tested induced a weight gain depression in the broilers (Tab. II). At day 21 PI, the average body weight of group 3 (inoculated with a cloned virus of GEI09 97M) was significantly higher than that of the other groups. At days 1, 2 and 4 PI, a few chicks of the infected groups showed weakness and depression. Macroscopically, most infected chicks had distended and pale small intestines with watery and mucous content from day 1 PI up to day 4 PI. Histological changes including vacuolar degeneration, ballooning and sloughing of the epithelium at the tip of the villi were present in all the infected groups (Fig. 1). The numbers of the affected chickens are presented in Table III. The lesions were present in all parts of the small intestine. However, the lesions were mostly present in the jejunum and ileum of all infected groups at days 1 and 2 PI. ARV NLI20 98M induced very quickly (day $1 \mathrm{PI}$ ),

Table III. Enteropathogenic effect of inoculation of 1-day-old SPF broilers with different avian reoviruses as measured by histological changes.

\begin{tabular}{lcccc}
\hline \multirow{2}{*}{$\begin{array}{l}\text { Group, } \\
\text { reovirus code (cluster) }\end{array}$} & \multicolumn{4}{c}{ Number of affected birds $(n=5)^{\mathrm{b}}$} \\
\cline { 2 - 5 } & day 1 PI & day 2 PI & Day 4 PI & day 7 \\
\hline 1 Control, PBS & 0 & 0 & 0 & 0 \\
2 GEI09 97M $(1,4,5)$ & 2 & 4 & 3 & 0 \\
3 GEI09 97M (4) & 4 & 3 & 2 & 0 \\
4 NLI19 97M(4) & 4 & 3 & 2 & 0 \\
5 NLI20 98M (2) & 5 & 2 & 3 & 0
\end{tabular}

\footnotetext{
${ }^{a}$ Reovirus code and cluster according to genotyping by Kant et al. [10].

Designation: $\mathrm{GE}=$ case in Germany; I = isolated at ID-Lelystad; $09=9$ th isolate of the year; $97=$ year of isolation; $\mathrm{M}=$ MAS originating. Furthermore: $\mathrm{NL}=$ The Netherlands; PBS = phosphate buffered saline.

${ }^{\mathrm{b}}$ Histological changes include vacuolar degeneration, ballooning and sloughing of the epithelium at the tip of the villi.
} 


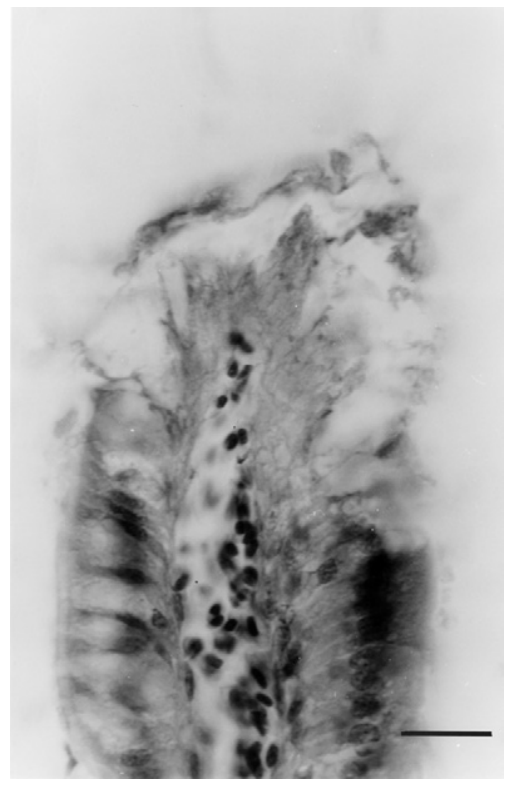

Figure 2. Apical denudation of the ileal villus from an SPF broiler infected with ARV NLI20 $98 \mathrm{M}$ at day 2 PI. Bar $=50 \mu \mathrm{m}$.

severe and widespread lesions like sloughing of the epithelium resulting in denuded villi (group 5) (Figs. 1 and 2). These lesions were more severe than in the other groups in trial 2. Moreover, this virus induced more severe early lesions in broilers than in White leghorn chickens. From day 7 PI on, the intestines of all ARVinfected groups appeared to be normal.

The epithelium of the bursa of Fabricius of all infected groups underwent vacuolar degeneration at days 4 and 7 PI whereas no histological changes occurred in the proventriculus, pancreas, or caecal tonsil in any of the infected groups of broilers. Also, at these time points, moderate lymphoid depletion was present in the bursa of Fabricius of broilers of group 5 (infected with ARV NLI20 98M).

Reovirus antigen was detected in the cytoplasm of enterocytes at the tip and middle section of the villi of the duode-

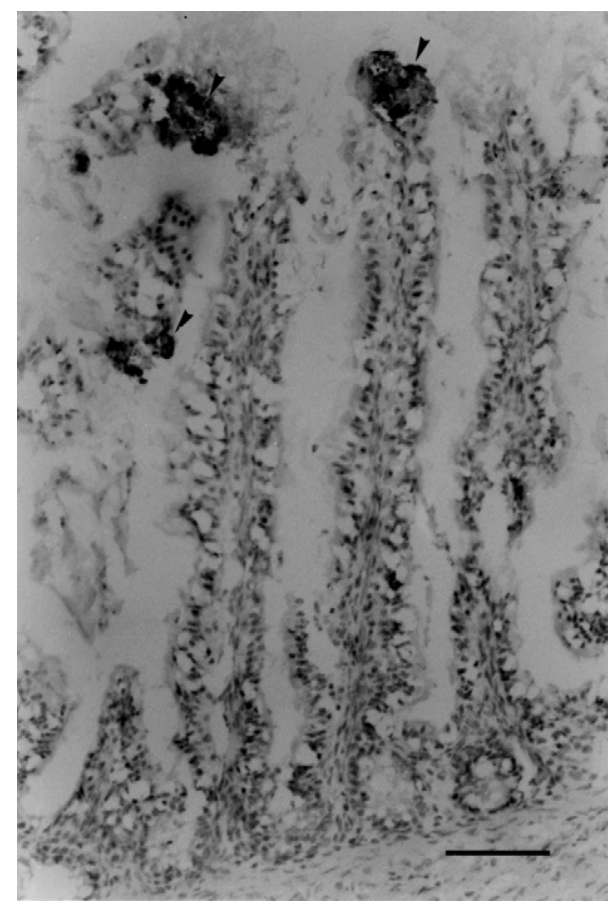

Figure 3. Immunohistochemistry. A reovirus antigen (arrow head) was detected at the tip and in the middle section of the jejunal villi of an SPF broiler infected with ARV NLI20 98 at day 2 PI. Bar $=105 \mu \mathrm{m}$.

num, jejunum, ileum, and in the caecal tonsil and bursa of Fabricius of all infected broiler groups at days 1, 2, and 7 PI (Fig. 3). The reovirus antigen was mostly found in the affected jejunum and ileum. From day 7 on, no reovirus antigen was detected in any of the infected groups. The control group did not have histopathological changes nor a positive reaction to the reovirus antigen.

The intestinal cellular immune response was studied in the jejunum of group 5 (infected with NLI20 98M) and in group 1 (control group). Macrophages/monocytes were present in the lamina propria of the jejunum of both groups and increased from day 4 PI on. At day 7 PI, macrophages/ monocytes $(\mathrm{pc} / \mathrm{sm})$ of the infected group were significantly higher than those of the 
Table IV. The effect of a MAS-associated reovirus infection in SPF broilers on the number of $\mathrm{CD}^{+} \mathrm{T}$-cells and macrophages/monocytes in the jejunum at day 7 and $14 \mathrm{PI}$.

\begin{tabular}{|c|c|c|c|c|}
\hline \multirow[t]{2}{*}{ Group } & \multicolumn{2}{|c|}{$\mathrm{CD}^{+}{ }^{+}$-cell ${ }^{\mathrm{A}}$} & \multicolumn{2}{|c|}{ Macrophage/monocyte ${ }^{\mathrm{A}}$} \\
\hline & day 7 PI & Day 14 PI & day 7 PI & day $14 \mathrm{PI}$ \\
\hline Control, PBS & $\begin{array}{c}0.14 \pm 0.10^{\mathrm{a}} \\
(45)^{\mathrm{B}}\end{array}$ & $\begin{array}{c}0.69 \pm 0.17^{\mathrm{a}} \\
(45)\end{array}$ & $\begin{array}{c}0.76 \pm 1.56^{\mathrm{a}} \\
(45)\end{array}$ & $\begin{array}{c}1.32 \pm 0.27^{\mathrm{a}} \\
(41)\end{array}$ \\
\hline NLI20 98M ${ }^{\mathrm{C}}$ & $\begin{array}{c}0.86 \pm 0.33^{\mathrm{b}} \\
(45)\end{array}$ & $\begin{array}{c}1.75 \pm 0.49^{\mathrm{b}} \\
(45)\end{array}$ & $\begin{array}{c}1.47 \pm 0.55^{\mathrm{b}} \\
(42)\end{array}$ & $\begin{array}{c}1.53 \pm 0.34^{\mathrm{a}} \\
(40)\end{array}$ \\
\hline $\begin{array}{l}{ }^{\mathrm{A}} \text { Number of C } \\
\text { villus }[\times 1000] \text {. } \\
{ }^{\mathrm{B}}()=\text { number } \\
{ }^{\mathrm{C}} \text { Designation: } \\
\text { year of isolatio } \\
\mathrm{a}, \mathrm{b} \text { Means } \pm \mathrm{SD}\end{array}$ & $\begin{array}{l}\text { lls or macrop } \\
\text { ations. } \\
\text { e Netherland } \\
\text { AAS originati } \\
\text { umn with no }\end{array}$ & $\begin{array}{l}\text { onocytes in } \\
\text { lated at ID- } \\
=\text { phosphat } \\
\text { superscript }\end{array}$ & $\begin{array}{l}\text { as positive ce } \\
\text {; } 20=20 \text { th is } \\
\text { gnificantly } \\
\text { gnime. }\end{array}$ & $\begin{array}{l}\text { quare micron } 0 \\
\text { the year; } 98= \\
\text {. }\end{array}$ \\
\hline
\end{tabular}

control group $(P<0.05)$. However, at day 4 (not shown) and 14 PI, there was no significant difference in macrophages/ monocytes $\mathrm{pc} / \mathrm{sm}$ between the infected and the control group. No $\mathrm{CD} 4^{+} \mathrm{T}$-cells were present in the jejunum of the control group at either time point studied (day 1, 2, 4, 7 and $14 \mathrm{PI})$, whereas few $\mathrm{CD}^{+}{ }^{+}$T-cells were present only at day 14 PI in the lamina propria of the jejunum of broilers of the infected group. From day 4 PI on, $\mathrm{CD} 8^{+} \mathrm{T}$-cells were present in the epithelium and lamina propria of the jejunum of both groups. The $\mathrm{CD}^{+}(\mathrm{pc} / \mathrm{sm})$ of the infected group was significantly higher than that of the control group from days 7 and 14 PI $(P<0.05)$ (Tab. IV).

\subsubsection{Virus re-isolation}

All cloacal swab samples from infected groups 2 to 5 were positive for reovirus isolation on day 7 PI. The swab samples from the control group were negative for reovirus isolation.

\subsubsection{Serology (trial 2)}

In the presera tested, no antibodies directed against the reovirus were detected. All sera obtained from the inoculated groups (2 to 5) at day 21 PI were positive against the reovirus. The control group had no antibodies to the reovirus.

\section{DISCUSSION}

All avian reoviruses isolated from the cases of malabsorption syndrome used in this study could induce lesions such as vacuolar degeneration and sloughing of enterocytes in the small intestine. Lesions were observed both in white leghorn chickens and in broilers. The lesions were, however, less severe in the white leghorn chickens. This was especially the case for NLI20 98M, which very quickly caused, in comparison to the other ARVs, severe lesions including apical denuded villi in the small intestines of the broilers. Jones et al. [9] have shown that broilers are more susceptible to tenosynovitis reoviruses than are white leghorns.

Although the ARVs caused intestinal lesions in the broilers, none of them caused weight gain depression. The lesions were mainly present at the tip of the villus, whereas the reovirus antigen was detected at the tip and middle part of the villi. The immunohistochemistry showing reovirus antigen has been previously shown by Jones et al. [8]. We speculate that ARVs preferably replicate in more differentiated enterocytes that are present at the tip of the villi. It is known that the villus epithelia are mostly generated from the crypt of Lieberkühn and move up along the villi. In this study, neither the lesion nor the reovirus antigen was present in the crypt of 
Lieberkühn. In MAS affected broilers, the intestinal lesions include enteritis with cystic formation of the crypt of Lieberkühn, villus atrophy and villus fusion [22]. This indicates that the reovirus alone cannot cause the same intestinal lesions as those found in MAS. Our results are in accordance with other studies that indicate that one cannot reproduce common features of MAS by using MAS-associated ARVs alone $[2,11]$. In contrast to our study, however, other investigators showed that reoviruses isolated from MAS chickens could induce weight gain depression [27, 28] with intestinal lesions [20] in broilers. On the contrary to our study, they did not use SPF broilers, but rather commercial broilers. In commercial broilers, the combination of the inoculated reovirus with agents already present in the broiler may cause the effect on weight gain.

Interestingly, at day $7 \mathrm{PI}$, lesions were still present in the infected groups of the white leghorns but not in the infected groups of broilers. The apical denuded villi observed in the infected white leghorn chickens at day 7 PI, could partly be an artefact caused by handling during collecting or processing of the tissues. We did not, however, observe this in the control white leghorns. On the contrary, it is likely that epithelial replacement occurs faster in broilers than in white leghorn chickens. Differences in the villus morphology and activities have been shown in that the villus epithelia develop more over the whole apical surface, have larger villi and wider microvilli [29] and show an earlier maturation in activities [30] in the broiler than in white leghorn chickens. Therefore, although the infected broilers had more severe intestinal lesions (at day 2 PI) because of the presence of more differentiated epithelia, the recovery might be faster due to a faster replacement of the epithelia.

Unexpectedly, at day 7 PI, all infected groups of broiler chickens were still positive to reovirus re-isolation although no lesions were observed at this time point.
The virus might be cleared with the sloughed cells resulting in the recovery of the affected villi when the chickens were older. Other mechanisms of clearance might also be involved. At day 7 PI, the number of $\mathrm{CD}^{+} \mathrm{T}$-cells and macrophage/ monocytes ( $\mathrm{ps} / \mathrm{sm})$ in the infected intestine had increased. An increased number of $\mathrm{CD} 8^{+} \mathrm{T}$-cells suggests a cytotoxic activity. Although we have no convincing data to support the role of these cells in the clearance of ARVS, others have also observed an increase in the number of $\mathrm{CD} 8^{+} \mathrm{T}$-cells [13], and T-helper 1 cells [4] in mice infected with reoviruses. Pertile et al. [18] suggest that macrophages play a role in the protection of cells by cytopathic effects of nitric oxide production on the host cell. Whether the activities of the immune cells can be correlated with a cell type-specific set of cytokines during a reovirus infection needs to be further studied. In this study, we did not examine the intestinal humoral immune response to reovirus infection. However, the reovirus-specific humoral immune response such as intestinal immunoglobulin $A$ and $G$ has been shown by another study [16].

We have described the reproduction of MAS by oral inoculation of homogenates obtained from the intestine of MAS affected broilers into 1-day old chicks [22]. In these affected broilers, we also found increased numbers of $\mathrm{CD} 8^{+} \mathrm{T}$-cells in the crypt of Lieberkühn that had early stages of cystic formation, especially in the jejunum (unpublished data). Therefore, we compared the $\mathrm{CD}^{+}{ }^{+} \mathrm{T}$-cells in the jejunum of one ARV infected broiler group with those in the control group. In the present study, the localisation of $\mathrm{CD}^{+} \mathrm{T}$-cells is rare in the crypt of Lieberkühn of the reovirus-infected broilers. Indeed, the MAS homogenates contained not only ARVs but also other agents and unknown substances. We hypothesise that MAS-associated reoviruses in combination with other viruses and /or bacteria in a MAS-homogenate cause the lesions and the localisation and increase of the $\mathrm{CD} 8^{+} \mathrm{T}$-cells in the small 
intestine. However, the role of the ARVs as a priming agent in the development of MAS lesions and weight gain depression needs to be further studied in combination with other agents or substances. This is currently under study.

\section{ACKNOWLEDGEMENTS}

We would like to thank Ralph Kok and Francis Balk for technical assistance and Guus Koch for critical reading of the manuscript.

\section{REFERENCES}

[1] Boot H.J., ter Huurne A.A.H.M., Peeters B.P., Gielkens A.L.J., Efficient rescue of infectious bursal disease virus from cloned cDNA: evidence for involvement of the 3'terminal sequence in genome replicaton, Virology 265 (1999) 330-341.

[2] Decaesstecker M., Charlier G., Meulemans G., Significance of parvoviruses, entero-like viruses and reoviruses in the aetiology of the chicken malabsorption syndrome, Avian Pathol. 15 (1986) 769-782.

[3] Dhillon A.S., Kibenge F.S.B., Page R.K., Viral arthritis in Fryers related to reovirus infection in breeders, Avian Dis. 30 (1986) 613-616.

[4] Fan J.Y., Boyce C.S., Cuff C.F., T-helper 1 and T-helper 2 cytokine responses in gutassociated lymphoid tissue following enteric reovirus infection, Cell. Immunol. 188 (1998) 55-63.

[5] Goodwin M.A., Davis J.F., McNulty M.S., Brown J., Player E.C., Enteritis (so-called runting stunting syndrome) in Georgia broiler chicks, Avian Pathol. 37 (1993) 451-458.

[6] Hieronymus D.R.K., Villegas P., Kleven S.H., Characteristics and pathogenicity of two avian reoviruses isolated from chickens with leg problems, Avian Dis. 27 (1983) 255-260

[7] Jeurissen S.H.M., Janse E.M., Ekino S., Nieuwenhuis P., Koch G., de Boer G.F., The monoclonal antibody CVI-ChN1-68.1 recognizes cells of the monocyte-macrophage lineage in chickens, Dev. Comp. Immunol. 12 (1988) 855-864.

[8] Jones R.C., Islam M.R., Kelly D.F., Early pathogenesis of experimental reovirus infection in chickens, Avian Pathol. 18 (1989) 239-253.
[9] Jones R.C., Kibenge F.S.B., Reovirus-induced tenosynovitis in chickens: The effect of breed, Avian Pathol. 13 (1984) 511-528.

[10] Kant A., Balk F., Born L., van Roozelaar D., Heijmans J., Gielkens A., ter Huurne A., Classification of Dutch and German avian reoviruses by sequencing the $\sigma \mathrm{C}$ protein, Vet. Res. 34 (2003) 203-212.

[11] Kouwenhoven B., Vertommen M., Goren E., Investigations into the role of reovirus in the malabsorption syndrome, Avian Pathol. 17 (1988) 879-892.

[12] Kouwenhoven B., Vertommen M., Van Eck J.H.H., Runting and leg weakness in broilers: involvement of infectious factors, Vet. Sci. Commun. 2 (1978) 253-259.

[13] London S.D., Cebra-Thomas J.A., Rubin D.H., Cebra J.J., CD8 lymphocyte subpopulations in peyer's patches induced by reovirus serotype 1 infection, J. Virol. 144 (1990) 3187-3194.

[14] Luna L.G., Haematoxylin-Eosin staining in: Manual of Histologic staining methods of the Armed Forces Institute of Pathology, 3rd ed., American Registry of pathology, New York, McGraw-Hill, 1968, pp. 32-37.

[15] Moradian A., Thorsen J., Julian R.J., Single and combined infections of specific-pathogen-free chickens with infectious bursal disease virus and an intestinal isolate of reovirus, Avian Dis. 34 (1990) 63-72.

[16] Mukiibi-Muka, G., Jones R.C., Local and systemic IgA and IgG responses of chicks to avian reoviruses: effects of age of chick, route of infection and virus strain, Avian Pathol. 28 (1999) 54-60.

[17] Ni Y., Kemp M.C.A., Comparative study of avian reovirus pathogenicity: virus spread and replication and induction of lesions, Avian Dis. 39 (1995) 554-566.

[18] Pertile T.L., Karaca K., Sharma J.M., Walser M.M., An antiviral effect of nitric oxide: Inhibition of reovirus replication, Avian Dis. 40 (1996) 342-348.

[19] Reece K.L., Frazier J.A., Infectious stunting syndrome of chickens in Great Britain: field and experiment studies, Avian Pathol. 19 (1990) 723-758.

[20] Rekik M.R., Silim A., Bernier G. Serological and pathogenic characterization of avian reoviruses isolated in Quebec, Avian Pathol. 20 (1991) 607-617.

[21] Rosenberger J.K., Sterner F.J., Botts S., Lee K.P., Margolin A., In vitro and in vivo characterization of avian reoviruses. I. Pathogenicity and antigenic relatedness of several avian reovirus isolates, Avian Dis. 33 (1989) 535-544. 
[22] Songserm Th., Pol J.M.A., van Roozelaar D., Kok G.L., Wagenaar F., ter Huurne A.A.H.M., A comparative study of the pathogenesis of malabsorption syndrome in broilers, Avian Dis. 44 (2000) 556-567.

[23] SPSS advance statistics ${ }^{\mathrm{TM}}$ version 6.1, SPSS Inc., Chicago, IL, 1994

[24] Tang K.N., Fletcher O.J., Villegas P., Comparative study of the pathogenicity of avian reoviruses, Avian Dis. 31 (1987) 557-583.

[25] Van der Heide L., Tenosynovitis/viral arthritis. A review, Avian Pathol. 6 (1977) 271284.

[26] Van der Heide L., Kalbac M., Infectious tenosynovitis (viral arthritis): characterization of a Connecticut viral isolant as a reovirus and evidence of viral egg transmission by reovirus-infected broiler breeders, Avian Dis. 19 (1975) 683-688.
[27] Van der Heide L., Lütticken D., Horzinek M., Isolation of avian reovirus as a possible etiologic agent of osteoporosis ("Brittle bone disease"; "Femoral head necrosis") in broiler chickens, Avian Dis. 25 (1981) 847-856.

[28] Vertommen M., van Eck J.H.H., Kouwenhoven B., van Kol N., Infectious stunting and leg weakness in broilers. I. Pathology and biochemical changes in blood plasma, Avian Pathol. 9 (1980) 133-142.

[29] Yamauchi K., Isshiki Y., Scanning electron microscopy observations on the intestinal villi in growing white leghorn and broiler chickens from 1 to 30 days of age, Br. Poult. Sci. 32 (1991) 67-78.

[30] Yamauchi K., Lida S., Isshiki Y., Posthatching development changes in the ultrastructure of the duodenal absorptive epithelial cells in 1, 10 and 60-d-old chicks with special references to mitochondria, Br. Poult. Sci. 33 (1992) 475-488. 